Horizons philosophiques

\title{
Les comités d'éthique comme mécanisme de contrôle des activités de recherche
}

Le point de vue de la philosophie

\section{Pierre Gendron}

Volume 5, numéro 2, printemps 1995

Le philosophe et l'état

URI : https://id.erudit.org/iderudit/800984ar

DOI : https://doi.org/10.7202/800984ar

Aller au sommaire du numéro

Éditeur(s)

Collège Édouard-Montpetit

ISSN

1181-9227 (imprimé)

1920-2954 (numérique)

Découvrir la revue

Citer cet article

Gendron, P. (1995). Les comités d'éthique comme mécanisme de contrôle des activités de recherche : le point de vue de la philosophie. Horizons philosophiques, 5(2), 109-131. https://doi.org/10.7202/800984ar d'utilisation que vous pouvez consulter en ligne. 


\section{LES COMITÉS D'ÉTHIQUE COMME MÉCANISME DE CONTRÔLE DES ACTIVITÉS DE RECHERCHE :}

\section{LE POINT DE VUE DE LA PHILOSOPHIE*}

La protection des personnes contre les risques de la recherche est le principal besoin qui a été à l'origine de la création des premiers comités d'éthique aux États-Unis. Le contexte qui les vit apparaître était en effet marqué par un conflit entre les exigences de la démarche scientifique, que traduisent les «normes de la recherche», et celles du traitement médical. Étaient en jeu, donc, non seulement l'intégrité physique et morale des personnes qui se prêtaient à la recherche médicale, mais également les intérêts des chercheurs, de la science et, finalement, de la société.

Mais la réalisation de cet objectif supposait la résolution d'un certain nombre de difficultés liées à la nécessité d'intégrer une forme d'évaluation scientifique au processus de l'évaluation proprement éthique, intégration nécessaire dans la mesure où les risques de la recherche faisant appel à des sujets humains

- Le présent article est issu d'une recherche que l'auteur a effectuée entre 1990 et 1993, alors qu'il était boursier post-doctoral à la Faculté de philosophie de I'Université Laval, dans le cadre d'une étude du processus décisionnel des comités d'éthique dans les hópitaux du Québec menée par le Groupe de recherche en éthique médicale (GREM). Ce projet a été réalisé grâce à une subvention du Conseil de recherche en sciences humaines du Canada. L'auteur remercie Fernande Bérubé-Genest, conseillère scientifique au Ministère de l'Industrie, du Commerce, de la Science et de la Technologie (Direction des politiques scientifiques), pour sa collaboration dans l'analyse du fonctionnement des comités d'éthique de la recherche; il remercie également Luc Bégin, Éric Gagnon, Bruno Leclerc et MarieHélène Parizeau, du GREM, pour leurs commentaires lors des révisions successives du document de travail qui a servi de base à la présente publication.

Une version abrégée de ce texte a été présentée le $1^{\text {er }}$ mars 1994 à la Faculté de droit de l'Université de Montréal dans le cadre des Conférences scientifiques du Centre de recherche en droit public. 
devaient être au moins compensés par la valeur du projet au plan scientifique.

II faut ajouter à cela les problèmes qu'entraîne la nécessité d'une représentation du public au sein des comités (suivant l'idée que le recours au processus démocratique assurera une meilleure protection des personnes), notamment ceux qui tiennent aux limites que rencontre tout effort pour favoriser la participation des profanes à la prise de décision dans les comités d'éthique de la recherche (étant admis que l'évaluation scientifique est d'abord une affaire de spécialistes).

Le but du présent article, par conséquent, sera de faire ressortir, en se fondant sur l'opposition classique entre pairs et profanes, une difficulté majeure dans le processus de mise en place des divers mécanismes de contrôle des activités de recherche.

\section{L'origine des comités d'éthique}

L'histoire des comités d'éthique montre à quel point les chercheurs ont toujours détesté que quelqu'un d'étranger ou du dehors vienne leur dire ce qu'il faut faire. Même le célèbre article intitulé "Ethics and Clinical Research", que Henry Beecher fit paraître en 1966 dans le New England Journal of Medicine, particulièrement accusateur à l'égard du milieu de la recherche, était d'abord destiné à un public de chercheurs et s'efforçait de parler leur langage.

Pourtant, comme l'a rappelé encore récemment David Rothman, quelques observateurs extérieurs au milieu médical acquirent assez tôt la conviction, partagée par quelques membres de la profession médicale, que quelque chose n'allait pas dans le monde de la recherche aux États-Unis, que la propension des chercheurs à faire valoir des arguments utilitaristes et leur parti-pris en faveur de certains intérêts les rendaient inaptes à se prononcer, au plan de l'éthique, sur l'expérimentation humaine. Bref, il n'était plus possible de compter sur le chercheur, à la différence du médecin traitant, pour qu'il agisse toujours dans le meilleur intérêt du patient devenu sujet d'expérimentation. C'est pourquoi le philosophe, 
le juriste et le législateur, qui ne s'étaient guère préoccupés dans le passé de ce qui se passait dans les laboratoires, se mirent à s'intéresser vivement à l'éthique de la recherche.

L'article de Beecher devint rapidement une référence, à l'extérieur aussi bien qu'à l'intérieur du milieu de la recherche, et contribua à lancer un mouvement qui introduisit de nouvelles règles du jeu et de nouveaux acteurs dans le processus décisionnel en médecine. La présentation par Beecher d'une vingtaine de cas d'abus évidents l'amenait à conclure que les procédés condamnables ou pour le moins discutables du point de vue de l'éthique n'étaient pas rares dans le domaine de la recherche. II fallut de toute urgence procéder à la mise en place de mécanismes assurant une meilleure régulation de la recherche faisant appel à des sujets humains. Si les exemples fournis par Beecher étaienten effetle moindrement représentatifs de la situation de la recherche, il aurait été imprudent de confier aux seuls chercheurs le soin de protéger les personnes qui participaient à leurs expériences. Une institution nouvelle figurait au cœur du système de régulation mis en place : le comité d'éthique de la recherche.

Mais ces changements de politique se limitèrent au début à confier le mandat d'évaluer l'éthique de la recherche à des comités de pairs; le chercheur n'était pas seul, mais les chercheurs pouvaient encore en 1966 régler entre eux leurs problèmes d'éthique. Durant la période de l'immédiat aprèsguerre, les chercheurs américains ne furent soumis à aucune contrainte venant de l'extérieur quant à la façon de diriger leur laboratoire. L'autonomie professionnelle dont ils jouissaient ne connaissait alors d'autres limites que celles imposées par les consciences individuelles, sans qu'interviennent vraiment les pouvoirs publics, les organismes subventionnaires ou même les établissements où se trouvaient les laboratoires. La protection des personnes demeurait la responsabilité du milieu de la recherche.

Selon Rothman, l'expérience de la deuxième Guerre mondiale avait transformé les attitudes et justifié par la suite jusqu'à un certain point certains comportements. Toute une 
génération de chercheurs, explique-t-il, avait été formée dans l'esprit d'un "combat" contre la maladie qui faisait partie intégrante de l'effort de guerre. Et cela expliquait également le type de "conscription" ${ }^{1}$ qui affectait particulièrement certaines catégories de sujets pressentis pour la recherche ${ }^{2}$.

Le public refusa toutefois au bout d'un certain temps de continuer à faire confiance aux chercheurs et cessa d'accepter tout simplement leurs rationalisations. Les chercheurs n'avaient certainement pas agi dans le meilleur intérêt de leurs sujets en injectant aux uns des cellules cancéreuses, à d'autres le virus de l'hépatite, ou en insérant des cathéters dans les cavités du cœur de certains patients. Certes, la réaction du public aurait pu être différente. En effet, si un médecin disait qu'il y avait dans telle expérience peu ou pas de risques, pourquoi ne pas le croire et penser comme lui que, de toute façon, la qualité de vie des sujets qui participaient à son expérience était pour ainsi dire inexistante et qu'ils avaient au moins la chance de pouvoir être ainsi encore utiles à l'humanité? Si un autre affirmait que les patients en institution qui participaient à une expérience sur telle maladie contagieuse l'auraient inévitablement contractée et qu'il était préférable pour eux d'être tout de suite suivis par son équipe qu'être soignés plus tard sur les étages, pourquoi ne pas le croire et penser comme lui que, de toute façon, ces sujets

1. «Nous devons nous rendre compte, disait déjà Hans Jonas, dans ses «Réflexions philosophiques sur l'expérimentation humaine", que le simple fait de faire appel à des volontaires, avec les pressions morales et sociales que cela entraîne inévitablement, équivaut, même avec une réglementation minutieuse du consentement, à une sorte de conscription, et implique nécessairement quelque sollicitation" (cf. Médecine et expérimentation, Québec, Presses de l'Université Laval, 1982, p. 322).

2. "It is no coincidence that this cohort of investigators [trained to think in utilitarian terms and ready to achieve the greatest good for the greatest number] took as their research subjects persons who were in one sense or another devalued and marginal: they were either retarded, institutionalized, senile, alcoholic, or poor, or they were military recruits, cannon fodder for battles in a war against disease" (David Rothman, Strangers at the Bedside : A History of How Law and Bioethics Transformed Medical Decision Making, New York, Basic Books, 1991, p. 80).

Sur l'histoire des comités d'éthique, voir aussi David Rothman, uHuman Experimentation and the Origins of Bioethics in the United States", Social Science Perspectives on Medical Ethics, Boston, Kluwer, 1990, p. 185-200. 
avaient au moins la chance de pouvoir être ainsi encore utiles à l'humanité? Mais les courants démocratiques, égalitaires et anti-autoritaires caractéristiques du mouvement des droits civiques finirent par exiger une réglementation plus stricte.

Cette description rejoint-elle la réalité québécoise et l'interprétation proposée par Rothman peut-elle s'appliquer au cas du Québec? Cela reste à vérifier; mais, en ce qui concerne les mécanismes de contrôle des activités de recherche, il est permis de faire l'hypothèse d'une continuité par rapport au modèle des premiers comités d'éthique aux États-Unis.

\section{Le «type idéal» du comité d'éthique}

Les résultats de l'observation méthodique des activités d'un comité d'éthique de la recherche, observation qui a été effectuée lors d'une enquête sur le terrain ${ }^{3}$, permettent de décrire ici, strictement pour les fins de l'analyse, le fonctionnement d'un comité-type ${ }^{4}$. Ce comité est composé d'une douzaine de membres et se trouve dans un hôpital universitaire reconnu comme établissement d'enseignement en sciences de la santé et doté d'un personnel, d'équipements spécialisés et des installations nécessaires pour faire de la recherche. Le comité relève du Conseil des médecins, dentistes et pharmaciens de l'hôpital (CMDP); son mandat officiel est de faire l'évaluation des projets de recherche faisant appel à des sujets humains et de déterminer si ces projets sont acceptables au plan de l'éthique. Pour accomplir cette tâche, le comité se rapportera aux lignes directrices du Conseil de recherches médicales du Canada.

Les avis du comité doivent être soumis au CMDP pour approbation finale; les recommandations faites aux chercheurs par le comité concernent surtout le formulaire de consentement; à l'occasion, il peut aussi demander une révision des protocoles

3. Ce travail d'observation a été effectué par Fernande Bérubé-Genest.

4. Le but ici n'est pas de porter un jugement de valeur sur ce que devrait être un comité d'éthique de la recherche, ni de répondre à des questions empiriques sur la réalité des comités d'éthique : tout au plus une telle description pourra-t-elle servir à déceler et à analyser les relations qui peuvent exister dans la réalité ainsi décrite. 
pour améliorer leur aspect scientifique. Mais l'emploi de la norme scientifique est en fait difficile à cerner; elle est rarement invoquée de façon explicite (bien que cela puisse arriver, surtout dans les cas où le protocole est refuse). Les membres du comité sont certes conscients du fait que l'évaluation de l'aspect scientifique des protocoles excède leur mandat officiel; mais ils n'en reconnaissent pas moins qu'il est nécessaire qu'un projet ait été validé au plan scientifique avant de pouvoir juger de sa qualité éthique. Il faut donc supposer un recours implicite aux normes scientifiques.

Les projets de recherche qui ont franchi avec succès les étapes de l'approbation par la direction du département concerné, puis de l'évaluation scientifique et éthique préalable par le président et deux autres membres du comité, sont discutés en comité. Le chercheur est alors invité à résumer son projet età répondre aux questions du comité. Si des modifications doivent être apportées, elles sont discutées avec le chercheur.

Le formulaire de consentement estensuite examiné. L'étude du formulaire de consentement est l'étape qui requiert généralement le plus de temps lors des réunions du comité d'éthique. Pour aider le chercheur et pour faciliter le travail d'évaluation, le comité propose une liste des points qui devront apparaître dans le formulaire. D'une part, le chercheur est invité à inclure un feuillet d'information expliquant pourquoi il est nécessaire de faire appel à des sujets humains, la raison d'être et les objectifs de l'étude, son déroulement (détails techniques à l'appui), les risques et effets secondaires, de même que les bénéfices escomptés (selon le cas, pour le sujet lui-même ou pour la société); d'autre part, le comité donne certaines indications relatives à l'engagement pris par le chercheur, qui doit fournir tous les détails concernant la collecte des données (dont la confidentialité, le consentement et, le cas échéant, les restrictions qui s'appliquent à certaines personnes) et les règles d'arrêt de l'étude (par retrait volontaire du sujet, sans préjudice, ou par décision du chercheur ou du médecin traitant, pour des raisons médicales ou autres). Si le projet est refusé, le chercheur est invité à réviser son protocole et à le présenter de nouveau 
à une prochaine réunion. Les protocoles acceptés par le comité sont acheminés par le président au CMDP pour approbation. Le chercheur attend alors l'avis confirmant qu'il peut commencer sa recherche.

Cependant, tout le monde ne sera pas rassuré pas ce travail à première vue excellent du comité d'éthique. Car il y a une difficulté : le comité, bien qu'il ait d'abord pour fonction d'assurer la protection des sujets participantà une recherche, se préoccupe en même temps de protéger le chercheur (et, par là, l'institution). La norme juridique, par conséquent, est un peu la rivale de la norme éthique. Elle est invoquée à la fois pour la protection du patient, du chercheur, et de l'institution. Elle apparaît surtout dans l'analyse du formulaire de consentement (avec la notion de risque). Enfin, elle explique peut-être aussi l'absence relative de suivi. La raison d'être du comité est, bien sûr, l'éthique; mais il y a également au sein du comité une volonté évidente d'aider le chercheur à réaliser son projet.

\section{La protection de l'autonomie professionnelle}

L'existence dans le fonctionnement du comité-type d'une volonté d'aider le chercheur (il faut supposer que cette volonté existe réellement, afin d'en examiner les conséquences logiques) exprime une tendance qui a aussi été observée lors d'une recherche faite par Éric Gagnon pour une thèse de doctorat en sociologie ${ }^{5}$. Les résultats de cette étude tendent à montrer que la volonté d'aider le chercheur à réaliser son projet est un facteur déterminant de la prise de décision dans les comités d'éthique de la recherche ${ }^{6}$. Cela n'est évidemment pas sans avoir certaines conséquences, notamment en ce qui concerne les conditions d'une véritable participation du public au travail des comités.

L'examen des procédures adoptées par certains comités

5. Les comités d'éthique pour la recherche au Québec: Institution et normalisation de la recherche médicale, École des Hautes Études en Sciences Sociales, Paris, 1993.

6. Certains résultats de cette recherche ont été publiés dans la revue Recherches sociographiques. 
d'éthique, par exemple, montre que «les classements, oppositions et distinctions qu'opèrent les comités entre les différents aspects des protocoles pour en faire l'évaluation éthique ont pour effet de limiter la portée de cette évaluation et de provoquer une dilution des responsabilités" ${ }^{7}$. C'est ainsi que l'examen de la question de la protection des personnes contre les risques de la recherche conduitinévitablementà se demander comment le fonctionnement des comités qui en sont chargés peut être affecté par la séparation entre l'évaluation éthique et l'évaluation scientifique.

\title{
Suivant Éric Gagnon :
}

L'évaluation de la qualité et de la pertinence scientifique par le comité d'éthique est loin de faire l'unanimité, pour la simple raison qu'on ne lui reconnaît pas la compétence pour la faire. En effet, bien qu'ils soient majoritairement des médecins, les membres des comités font rarement partie de la spécialité dont relèvent les projets examinés, et ne sont pas jugés aptes par les chercheurs à décider de leur qualité et leur contribution à l'avancement de la recherch $\theta^{8}$.

Il y a donc là une difficulté dont l'importance ne saurait être minimisée. À cet égard, la place qu'occupe l'examen du formulaire de consentement semble tout à fait symptomatique :

\begin{abstract}
Si le comité ne discute pas uniquement du consentement, s'il interroge le chercheur sur le choix des méthodes, l'intérêt de son étude, la nécessité de réaliser tel ou tel test, c'est sur la formule de consentement malgré tout qu'il peut agir le plus efficacement. [...] Le comité évitera ainsi les questions pour lesquelles des corrections techniques sont insuffisantes et qui risquent de remettre en cause le projet; il contournera les questions éthiques, les dilemmes, et, quand il s'en présente un, il s'efforcera de le ramener à un problème technique. La situation sera réinterprétée de façon qu'elle n'appelle plus un choix de valeurs?
\end{abstract}

7. Cf. Éric Gagnon, «Les comités d'éthique pour la recherche comme entreprise d'interprétation», Recherches sociographiques, 32 (1991), p.221.

8. Ibid., p. 224.

9. Ibld., p. 230. 
Cette critique occupe une position centrale parmi celles qui peuvent être adressées aux comités d'éthique de la recherche, car elle permet d'établir un lien avec les exigences du développement «technoscientifique». Les comités d'éthique de la recherche semblent aux yeux de plusieurs se soucier davantage de la forme du consentement que de sa mise en œuvre, d'où l'absence relative de suivi par les comités et la persistance d'un doute quant au rôle effectif du membre externe :

Le comité d'éthique de la recherche peut être critiqué à plusieurs égards. [...] Actuellement, par sa composition dominée par des médecins et des scientifiques, un tel comité s'appuie sur un consensus préalable qui va dans le sens du développement technoscientifique. Le représentant du public ou le membre extérieurà l'hôpital est généralement minoritaire et ne peut guère, en pratique, s'opposer à une recherche en invoquant, par exemple, sa pertinence sociale ${ }^{10}$.

La question du suivi oblige donc en quelque sorte à élargir la problématique de la protection des personnes, à s'interroger sur l'étendue des responsabilités du comité d'éthique de la recherche. Le comité doit-il se préoccuper de la protection des sujets de recherche jusque dans le déroulement d'une expérience? Doit-il assumer l'évaluation scientifique des protocoles? Bref, jusqu'où doit-il étendre son contrôle?

\section{Les normes de la recherche}

La protection contre les risques de la recherche, dans la mesure où elle exige un suivi au plan scientifique de la part des comités d'éthique, implique un minimum de compétence pour juger des questions techniques. Cela dit, quel rôle l'évaluation scientifique joue-t-elle exactement dans le processus de l'évaluation proprement éthique?

\section{a) La science et la technique scientifique}

L'activité scientifique a pour but l'acquisition des connaissances. Les normes de la recherche sont constituées

10. Cf. Marie-Hélène Parizeau, "Comités d'éthique", Les mots de la bioéthique, Bruxelles, De Boek-Wesmael, 1993, p. 73. 
par tout ce qui, dans cette activité, sertà déterminer le choix des démarches sur la base desquelles les résultats d'une recherche peuvent bénéficier du statut de connaissance scientifique. L'entreprise scientifique, en fait, se caractérise par le type de contrôle des démarches que de telles normes permettent d'assurer; car la science progresse en se donnant certains moyens de connaissance. Mais la science fournit également des moyens de connaissance aux autres domaines d'activité (comme la médecine). C'est pourquoi l'expression «norme du savoir scientifique" peut vouloir dire deux choses: elle signifie d'abord la totalité des critères qui permettent de juger du caractère scientifique d'un savoir ou d'un ensemble de connaissances; mais elle exprime aussi le fait que les connaissances scientifiques en général peuvent se voir attribuer une valeur normative. Le savoir scientifique peut alors être utilisé en tant qu'argument pour convaincre, dans une discussion ou au cours d'une délibération, par exemple dans un comité d'éthique. Certes, cet usage particulier est lui-même fondé sur l'utilisation technique possible du savoir scientifique et suppose, par conséquent, la possibilité de poser en général et de résoudre de purs problèmes techniques ${ }^{11}$. Mais n'importe quel élément normatif du discours scientifique peut théoriquement intervenir dans la prise de décision, en dehors du contexte technique proprement dit, et s'intégrer à la discussion entre membres d'un comité d'éthique.

Les critères qui constituent la norme du savoir scientifique sont potentiellement innombrables (aussi bien à cause de la complexité des problèmes qu'à cause de la diversité des formes que peut prendre ce savoir). La simple observation d'un

11. II s'agit des problèmes qui concernent uniquement le choix (effectué d'après les lois de l'expérience) des moyens appropriés pour atteindre une fin donnée; car «là où il est possible de définir d'une façon absolument univoque la fin, nous avons affaire à une simple inversion d'une relation causale, et par conséquentà un pur problème "technique" (Max Weber, Essais sur la théorie de la science, Paris, Plon, 1965, p. 473). Et c'est parce que la science apporte une solution à des problèmes de ce type que le travail scientifique peut être considéré comme solidaire d'un "progrès" et qu'un contenu précis peut être donné à cette notion de progrès (cf. Max Weber, Le savant et le politique, Paris, Plon, 1959, p. 75). 
phénomène, la description d'une réalité perceptiblevisuellement, doit déjà répondre à de nombreux critères. Le travail d'observation doit permettre de comparer certains faits (établis suivant les conventions propres à chaque discipline); il doit par conséquent répondre à des exigences relatives à la quantité, à la variété et à la durée dans le temps. Pour un meilleur contrôle des démarches, la «reproduction» des résultats doitêtre possible dans les conditions décrites et fournies en principe dans le "protocole» d'une expérience. Le travail théorique est luimême soumis à des normes relatives à la cohérence logique interne, au degré de formalisation, à l'établissement de relations causales, à la vérification des hypothèses, aux types de régularités observables et à la validité des échantillons; mais aussi à des normes qui permettent une évaluation comparée des théories comme la généralité et la simplicité. Mais c'est l'objectivité de la connaissance qui est sans doute l'expression la plus universellement reconnue comme l'idéal de la science. Elle se fonde sur l'application du principe de la "neutralité axiologique", qui consiste en l'abstention systématique de tout jugement de valeur dans l'établissement des faits (ce qui toutefois ne signifie aucunement l'absence de rapport aux valeurs, car il n'y a pas de science sans présuppositions).

Plus généralement, la norme peut se définir comme ce qui doit être (suivant un idéal, un modèle, ou une règle ${ }^{12}$ ), ou bien

12. La norme et la règle sont des notions qui peuvent s'éclairer mutuellement. Comme l'explique Julien Freund dans son Introduction aux Essais sur la théorie de la science de Max Weber : "La notion de règle [d'après l'analyse qu'en a faite Max Weber] comporte deux significations fondamentales. En premier lieu elle désigne des énoncés généraux portant sur des connexions causales relatives à l'être [...]. En second lieu elle désigne une norme d'après laquelle on mesure au nom d'un jugement de valeur les événements passés, présents et futurs. [...]

Il existe cependant d'autres sens qui ne se laissent pas réduire sans plus à l'un des deux précédents, par exemple les règles que l'on appelle les maximes de l'activité. Prenons l'exemple de Robinson [...]. Le héros du roman de Defoe menait malgré tout dans sa solitude une vie économique rationnelle, compte tenu des conditions de son existence, puisqu'il soumettait la consommation des biens et le bénéfice qu'il espérait tirer de son travail (ne faisait-il pas des réserves de semences et ne marquait-ll pas les arbres à abattre au cours de l'hiver suivant?) à des règles, et précisément à des règles économiques. [...] Cette solitude absolue, en dehors de tout contact avec l'autre, est un comportement qui relève de la pure technique" (op. cit., p. 80-85). 
comme ce qui est conforme à la majorité des cas, ou à l'usage (comme en linguistique), ou ce qui correspond à l'état habituel. L'opposition entre le fait et la norme est donc relative. C'est d'abord en tant qu'institution que la science est régie par des "normes» destinées à garantir l'objectivité, la rationalité et le caractère cumulatif des résultats qui sont le fruit de ses démarches. Pour le sociologue des sciences, il existe certaines "règles du jeu», dont l'ensemble constitue une "normativité", ou un "éthos", qui s'impose sous peine de sanctions diverses aux acteurs sociaux que sont les scientifiques ${ }^{13}$.

b) L'éthos professionnel des chercheurs scientifiques

Il y a donc, en science, valorisation de certaines procédures et de certains comportements comme étant conformes àl'éthique ou plutôt à «l'éthos professionnel» des scientifiques, qui peut être assimilé à un ordre normatif intériorisé, et qui correspond à l'ensemble de règles suivies par le chercheur dans la conduite de sa vie professionnelle. Il en résulte un ensemble de normes socio-professionnelles qui forment pour ainsi dire «l'armature éthique" du système normatif propre à l'institution scientifique. Cette structure comprend quatre composantes principales (les fameuses «normes de Merton»).

Premièrement, la communication des découvertes et le partage des connaissances est obligatoire pour qu'elles puissent être considérées comme faisant partie de la science. Les résultats de la recherche doivent avoir un caractère public et par conséquent doivent faire l'objet d'une mise en commun. La science est le produit d'un effort collectif; seule la «propriété intellectuelle" est attribuable, à des fins de reconnaissance

13. "The ethos of science refers to an emotionally-toned complex of rules, beliefs, values, and presuppositions which are held to be binding upon the scientist. Some phases of this complex may be methodologically desirable, but observance of the rules is not dictated solely by methodological considerations. This ethos, as sole code generally, is sustained by the sentiments of those to whom it applies. Transgression is curbed by internalized prohibitions and by disapproving emotional reactions which are mobilized by the supporters of the ethos. Once given an effective ethos of this type, resentment, scorn, and other attitudes of antipathy operate almost automatically to stabilize the existing structurew (Robert K. Merton, "The Normative Structure of Science", The Sociology of Science, Chicago, The University of Chicago Press, 1973, p. 258). 
sociale, aux individus.

Deuxièmement, un certain universalisme est constitutif de l'idéal de la science. La validité universelle des résultats de la recherche tient au fait que certaines démarches sont adoptées (théoriquement parce qu'elles sont les plus rigoureuses) par tous les scientifiques dans leur travail d'observation et d'interprétation des résultats. Aucune source ne devrait être négligée ou minimisée, et la valeur des arguments en faveur d'une démonstration ne devrait nullement être obscurcie par des considérations de nationalité, de race, de religion, d'ancienneté ou d'appartenance à un sexe ou à une classe sociale. Troisièmement, un certain désintéressement est attendu des chercheurs, c'est-à-dire le renoncement de principe à toute motivation extra-scientifique, comme l'attrait du pouvoir et de l'argent.

Quatrièmement, un certain scepticisme de méthode est de rigueur en science. Cela signifie que tout doit pouvoir être mis en doute aux yeux des scientifiques et soumis par eux à l'analyse (ce qui peut paraître menaçant pour les institutions autres que la science).

Les normes techniques, quant à elles, concernent les aspects de la recherche qui sont les plus étroitement liés à la démarche expérimentale; elles ont un caractère plus «local». Elles peuvent rapidement se multiplier et peuvent varier suivant l'évolution normale des disciplines, telle que celle-ci se reflète dans le contenu des publications savantes, et suivant les progrès de l'instrumentation; elles interviennent dans l'évaluation lorsqu'il est possible de constater empiriquement l'adéquation des moyens à une fin donnée. Mais les arbres ne doivent pas cacher la forêt: cette diversité ne doit pas faire oublier l'existence d'une pratique de la science dite normale, dont le modèle risque de s'imposer dans tous les domaines où les scientifiques peuvent exercer une influence. C'est sans doute à ce type d'influence qu'il faut attribuer la tendance générale observée par Éric Gagnon suivant laquelle les comités cherchent à réduire les questions d'éthique et à les transformer en questions "techniques", plus facile à résoudre parce que davantage 
"maîtrisables» et plus proches de la démarche de résolution de problèmes qui est déjà familière aux membres de ces comités.

Mais la relation qui existe entre l'évaluation scientifique et l'évaluation éthique de la recherche concerne des enjeux plus vastes qu'une amélioration souhaitable des procédures. Pour porter un jugement sur la valeur scientifique (et non pas se contenter de simplement valider un projet), il est nécessaire pour les comités d'éthique d'accéder à une démarche de type réflexif, et cela pour au moins deux raisons. D'une part, la réflexivité de la démarche des comités est nécessaire parce que le jugement éthique, en tant que tel, comme l'explique Jean Ladrière, transcende l'ordre des faits ${ }^{14}$. D'autre part, le jugement éthique doit faire appel à la réflexion à cause du caractère provisoire de la norme ${ }^{15}$.

Par conséquent il faudrait que les membres des comités d'éthique soient en mesure de porter ultimement un jugement critique sur la science; et pour cela, comment pourraient-ils se dispenser de l'obligation de se faire une idée assez précise de l'impact que la science peut avoir sur l'éthique?

\section{C) L'impact de la science}

Des situations nouvelles sont créées non seulement du fait que les progrès de la science et de la technologie entraînent l'apparition de nouveaux problèmes, mais aussi parce qu'elles suscitent, parun phénomène d' «induction", de nouvelles valeurs; la science et la technologie peuvent donc avoir un impact sur l'éthique dans la mesure où les valeurs spécifiques qu'elles

14. «Un fait, par lui me̊me et en tant que tel, ne contient aucune indication de valeur. La reconnaissance éventuelle du caractère éthique d'un état de choses ne peut venir que d'une démarche qui prend son point de départ dans la prise de conscience des exigences propres à l'ordre éthique. La reconnaissance de cet ordre en tant que tel et de sa spécificité précède nécessairement l'attribution d'une valeur éthique à tel ou tel état de choses" (Jean Ladrière, "Biologie et morale", La bioéthique, Québec, Presses de l'Université Laval, 1979, p. 64).

15. "Les normes constituées sont comme des points d'appul pour l'invention éthique, parce qu'il est de la nature de la norme, en tant qu'elle est médiation particularisante de l'exigence éthique comme telle, de transcender les types mêmes de situations auxquels elle se réfère explicitement et de porter ainsi une visée susceptible de donner lieu, dans de nouveaux contextes, à de nouvelles thématisations" (ibid., p. 76). 
diffusent dans l'ensemble de la culture peuvent être considérées comme ayant une portée éthique. Elles peuvent donc constituer pour l'éthique un facteur d'évolution. Comme l'explique encore Ladrière :

Les variations de la normativité éthique, amplement attestées par l'histoire des cultures, suffisent déjà à faire voir que les critères concrets de l'éthique ne sont nullement donnés a priori mais qu'il y a une historicité de l'éthique, c'est-à-dire une émergence progressive des normes, et donc une véritable créativité axiologique. L'intervention de la science et de la technologie dans le domaine éthique ne fait que souligner davantage ce point de vue et, de plus, permet de saisir de façon plus concrète et plus précise comment peut s'effectuer la détermination des normes ${ }^{16}$.

Cependant, cette circonstance comporte aussi le risque d'une fermeture de la science sur elle-même :

Dansla mesure où la science nous fournit une connaissance de plus en plus précise et étendue du fonctionnement des systèmes naturels et artificiels, on pourrait considérer qu'elle est à même de suggérer des indications précises, de caractère normatif, pour l'action. [...] Ce serait alors la science elle-même qui serait le principe justificateur du système des normes. Ou, plus exactement, ce serait la valeur immanente constitutive de la science [la connaissance dite objective] qui constituerait la valeur fondamentale et serait à la fois instauratrice et justificatrice à l'égard de tout le système des normes éthiques ${ }^{17}$.

L'histoire récente de l'expérimentation humaine en médecine, le scandale provoqué par les révélations de Henry Beecher aux États-Unis et la crise d'autorité qui s'est étendue ensuite du laboratoire de recherche au cabinet médical témoignent de la nécessité de prendre une distance critique à l'égard de toutes les formes d'auto-régulation. Le problème que pose la nécessité d'une représentation du public et d'une véritable participation des profanes au processus décisionnel des comités d'éthique trouve ici son point d'articulation.

16. Cf. Jean Ladrière, Les enjeux de la rationalité, Paris, Aubier-Montaigne, 1977, p. 155.

17. Ibid., p. 147-148. 


\section{La représentation problématique du public}

Le sociologue Talcott Parsons, spécialiste des questions relatives à l'exercice de la profession médicale, s'est intéressé au phénomène nouveau que représentait, en 1969, la formation des premiers comités d'éthique de la recherche. Dans une brève analyse de la fonction sociale de la recherche scientifique, il a cherché à montrer comment l'étude des normes scientifiques intervenait dans la compréhension des rapports de pouvoir entre les chercheurs professionnels et les profanes, qu'il s'agisse des sujets qui acceptent de participer à une recherche en signant un formulaire de consentement ou bien des représentants du public qui doivent approuver un protocole en tant que membres profanes d'un comité d'éthique.

Or, c'est le statut de "pair», implicitement revendiqué dans toute évaluation scientifique, qui fait problème dans la participation des profanes au travail des comités d'éthique et c'est ce qui permet de mettre en évidence le type de "décalage» qui caractérise les rapports de pouvoir qui impliquent l'autorité des scientifiques et des experts, dans la recherche sur des sujets humains. D'entrée de jeu, comme chez Merton, la question est abordée par Talcott Parsons du point de vue de la norme professionnelle et en fonction de l'autonomie institutionnelle de la science. L'éthique est pour lui d'abord un problème de régulation sociale. Certes, la confiance du public devrait être la contrepartie de l'intégrité scientifique garantie en principe par le jugement des pairs. Mais cette confiance étaitelle méritée? L'histoire des comités d'éthique de la recherche montre que certains ont eu dans le passé de bonnes raisons d'en douter. C'est pourquoi il a fallu retrouver une confiance qui avait été perdue; les comités étaient là pour accomplir cette fonction dans l'intérêt même de la science et donc, selon Parsons, pour le bien de la société ${ }^{18}$.

18. "The inclusion of the ulay" element in the positive functioning of the professional complex is the primary mechanism by which [the] tendency to withdraw or implement the right to resign is counteracted, and the basis of trust preserved and strenghtened. The positive participation of the research subject and its ethical regulation hence are of special salient significance, because research has become the most important spearhead of the trend of progressive advance of modern 
De ce point de vue, les comités pouvaient donc sembler avoir pour fonction d'accomplir une sorte de «rituel», pour reprendre l'expression de François Isambert. En effet, au moment même où les grands pourvoyeurs de fonds de la recherche médicale mettaient sur pied un contrôle éthique en même temps que scientifique des recherches qu'ils subventionnaient s'établissait, comme l'explique Isambert, «un complexe éthico-méthodologique que l'on serait tenté d'appeler métaphoriquement un «rituel» destiné à pourvoir l'essai clinique d'un ensemble de conditions légitimantes formant une sorte de rempart contre les possibles craintes ou indignations d'un public plus prompt à s'assimiler à des victimes de sacrifices humains qu'aux consommateurs protégés de thérapeutiques rigoureusement éprouvées ${ }^{19}$ ".

Plus les comités auront tendance à fonctionner sur le mode de la «régulation sociale", plus certaines aptitudes à la réflexion seront nécessaires. Or, cette la tendance des comités d'éthique à fonctionner sur le mode de la régulation a sans doute son origine dans le type de rationalité que les comités mettent en œuvre lorsqu'ils se donnent certaines règles ${ }^{20}$. Si la signification des règles en général est de servir comme instrument d'un pouvoir ${ }^{21}$, David Rothman a peut-être raison de penser que

societies" (Talcott Parsons, "Research with Human Subjects and the "Professional Complex" ", Action Theory and the Human Condition, New York, Free Press, 1978, p.64).

19. Cf. François-André Isambert, "L'expérimentation sur l'homme comme pratique et comme représentation", Actes de la recherche en sciences sociales, 68 (1987), p. 28.

20. Commel'explique Frederick Schauer: "Rule-based decision-making is premised in part on the belief that none of us, ordinary or not, have the mental capacity incessantly to consider all of the things that an "all things considered" decisionmaking model requires of us. If we are not to be paralysed by uncertainty, and stumble into numerous errors just because we have too little time to consider too much, we must often simplify our thought processes, using a form of decision making that limits us to the consideration of a manageable array of factors. [...] Rules also serve this agenda-simplifying or desk-clearing function, taking much off the agenda so that what remains can be dealt with the care and detail it deserves. In this respect, rules have silent virtues" (cf. Playing by the Rules: $A$ Philosophical Examination of Ruled-Based Decision Making in Law and in Life, Oxford, Clarendon Press, 1991, p. 229-230).

21. «Rules, telling agents what they should do and thus what they should not, are the implements by which roles are established and power is allocated" (ibid., p. 232). 
l'éthique médicale (en faisant éventuellement cause commune avec le droit) ne peut faire mieux que d'opposer à l'autorité des médecins et des chercheurs de nouvelles formes d'autorité22.

Or, la participation des "profanes" à ces nouvelles formes d'autorité et à l'activité décisionnelle des comités d'éthique est solidaire d'une dynamique sociale extrêmement complexe (qui implique notamment le type de rationalité qui est propre à la gestion des organisations), dynamique sur laquelle il convient de s'attarder encore un peu avant de conclure.

\section{Les comités d'éthique à l'hôpital}

Une étude de Guy Rocher a déjà fourni une analyse de la dynamique propre au contexte dans lequel s'effectue le travail de certains comités d'éthique. Suivant cette étude, il ne faudrait pas s'imaginer que le fonctionnement sur le mode régulateur est le fait des comités d'éthique en tant que tels et d'eux seuls; c'est tout le domaine de la bioéthique qui peut être vu comme un processus de régulation sociale. Comme l'explique Rocher :

La bioéthique se trouve dans une situation ambiguë. D'une part, elle est apparue et s'est élaborée parce que certains aspects de la modernité l'appelaient et l'interpellaient [notammentl'hégémonie de la technoscience]. Parailleurs, certains autres aspects de la même modernité [dont la rationalité économique] imposent aux porteurs de la bioéthique de faire face à une rude concurrence pour se faire entendre et se faire accepter ${ }^{23}$.

Les liens qui existent entre régulation et rationalisation expliqueraient pourquoi l'esprit critique qui caractérise le mode

22. «The transformations in medical decision making, as vital as they are, have come with a price. To alter the balances between doctor and patient and medicine and society encouraged, unavoidably, the intervention of a greater number of third parties. Ironically, to cope with the doctor as stranger and the hospital as strange, to respond to perceived conflicts of interest and to the power of new technologies, it appeared necessary to bring still more strangers to the bedside. [...] Constraining one authority figure required creating other authority figures» (Rothman, Strangers at the Bedside, op. cit., p. 260).

23. Guy Rocher, "La bioéthique comme processus de régulation sociale: le point de vue de la sociologien, Bioéthique: méthodes et fondements, Montréal, Association canadienne-française pour l'avancement des sciences, 1989, p.55. 
réflexif de fonctionnement peut être perçu comme une menace à la paix institutionnelle qui règne dans l'hôpital et donc comme un facteur de "désordre».

La croissance des coûts de la pratique médicale, envisagée dans une perspective de gestion des ressources rares ou limitées, introduit rapidement la logique de la rationalité économique, qui entre ainsi en concurrence avec la bioéthique. Or, dans une telle concurrence, comme l'explique Guy Rocher, le poids de la bureaucratie est déterminant; elle peut faire pencher la balance: la normativité émerge des rapports de pouvoir. "Si l'on veut comprendre le fonctionnement d'un hôpital, dit-il, la normativité interne qu'il s'est faite, il faut tenir compte des groupes professionnels porteurs d'intérêts différents, du pouvoir réel de chacun, de son statut et de son rôle, des rapports qu'il entretient avec d'autres groupes. II faut aussi localiser les «poids lourds" dans la prise de décision"24».

La réflexion, le passage des comités d'éthique à un mode réflexif de fonctionnement, est donc un préalable essentiel à toute véritable prise en compte des considérations éthiques. Car aussi longtemps que les comités fonctionneront sur un mode simplement régulateur, fût-ce pour exercer une fonction utile d'aide à la décision, la bioéthique risque de se réduire à une technique au service du consensus. La recherche de consensus produit alors «des normes, des règles qui assurent une certaine paix dans l'hôpital, sans qu'il soit nécessaire de poser les problèmes en termes éthiques ${ }^{25}$ ".

En fait, l'idée d'une norme éthique qui serait en situation de "concurrence" avec les autres normes (avec lesquelles, par conséquent, elle ferait nombre) n'a peut être vraiment de sens que du point de vue de la "valeur de succès" de l'action qui se détermine d'après des normes. Or, dans ce type de concurrence, qui tend à se généraliser au nom de la science et de la technique scientifique, une conclusion semble inéluctable: la norme éthique ne fait pas le poids. 
Faut-il donc se résigner à voir la mentalité utilitariste régner sans partage à l'hôpital? Ce serait oublier qu'à côté de la "valeur de succès" d'une action il y a aussi, comme le faisait remarquer Max Weber, la "valeur de conviction".

Mais la tâche première des comités d'éthique n'est-elle pas de réparer ce genre d'oubli?

\section{Conclusion : La vie des normes}

Ce qui précède montre à quel point s'impose, dans la réflexion actuelle sur le travail des comités d'éthique, le thème de la violence.

Les débuts de la bioéthique et la naissance des premiers comités d'éthique de la recherche eurent lieu dans un contexte marqué par la violence. Les normes de la recherche ellesmêmes, qu'elles soient de type socio-professionnel ou qu'elles soient purement techniques, sont potentiellement génératrices de violence; leur application peut aussi bien ajouter aux risques de la recherche que servir à limiter ces risques. C'est ce qui apparaît d'emblée dans l'examen des problèmes relatifs à la protection des personnes, qui se compliquent dans la mesure où existe dans les comités d'éthique de la recherche une volonté d'aider le chercheur à réaliser son projet.

La norme éthique vise le bien des personnes; et s'il ne s'agissait que d'assurer leur protection contre les risques bien identifiés de la recherche, la tâche des comités serait assez simple. Mais à partir du moment où les normes de la recherche, qui visent d'abord le bien de la science (quelles que soient les prises de position qui peuvent exister dans la société sur la valeur de la science et sur ses finalités), commencentà intervenir dans le travail d'évaluation fait par les comités, la fonction de "protection" qu'ils assurent implique beaucoup de choses et beaucoup de monde.

Le problème n'est pas que les comités puissent exercer une fonction d'aide à la décision, donc que leurs membres fassent figure d'experts, ou même qu'ils appuient le chercheur dans la réalisation de son projet; le problème vient de l'utilisation possible des personnes "uniquement comme un moyen". 
C'est lorsque cela arrive qu'il faut parler de violence.

La professionnalisation de l'activité scientifique a créé des risques nouveaux, dont certains sont propres à la recherche faisant appel à des sujets humains, et a ainsi rendu nécessaire une plus grande vigilance de la collectivité. En même temps, cette professionnalisation rend difficile la participation du public profane (c'est-à-dire non spécialisé) au travail des comités qui ont pour mandat d'exercer une telle vigilance, à cause de l'obstacle que représente le statut de pair exigé par l'évaluation scientifique.

En outre, la relation qui existe entre les membres d'un comité appartient pour une bonne part à l'ordre de la communication. D'où la nécessité de voir aussi le type de risque qu'impliquent la maîtrise du langage et la possibilité de maîtriser l'autre par le langage. Ainsi, l'impératif du consensus peut empêcher l'émergence d'une parole, par exemple en produisant ce jargon des spécialistes qui s'apparente au phénomène de la «langue de bois".

\section{L'éthique est-elle une espèce menacée?}

La raison pour laquelle la norme éthique serait «menacée» est finalement facile à comprendre. Car sa mise en œuvre, dans l'attribution d'une valeur éthique à tel ou tel état de choses, suppose, comme le disait Jean Ladrière, la reconnaissance d'un ordre éthique et des exigences propres à cet ordre. Cela suppose, comme il a été vu, qu'il y ait une valeur de conviction à côté de la valeur de succès; et cela peut déranger. Comme l'explique Guy Rocher, à propos des normes et des règles qui assurent la paix dans l'organisation hospitalière, la réflexion éthique risque de compromettre une paix difficilement acquise et qu'il faut protéger contre tout facteur de "désordre», contre tout ce qui peut la menacer, "y compris les considérations éthiques» ${ }^{26}$.

Quant à la tendance observée dans les comités, où les problèmes d'éthique sont parfois ramenés à des problèmes techniques, elle va dans le même sens; elle peut s'expliquer aussi par cette «rationalisation intellectualiste que nous devons 26. Ibid. 
à la science et à la technique scientifique ${ }^{27}$ ". Or, il est difficile de trouver un meilleur exemple que celui de la médecine moderne (qui compte parmi les technologies les plus développées du point de vue scientifique) pour faire comprendre la signification profonde d'une telle rationalisation :

\begin{abstract}
Exprimée de façon triviale, la "présupposition" générale de l'entreprise médicale se présente ainsi: le devoir du médecin consiste dans l'obligation de conserver la vie purement et simplement et de diminuer autant que possible la souffrance. [...] Mais la médecine ne pose pas la question si la vie mérite d'être vécue et dans quelles conditions. Toutes les sciences de la nature nous donnent une réponse à la question: que devons-nous faire si nous voulons être techniquement maîtres de la vie? Quant aux questions: cela a-t-il au fond et en fin de compte un sens? devonsnous et voulons-nous être techniquement maîtres de la vie? elles les laissent en suspens ou bien les présupposent en fonction de leur but ${ }^{28}$.
\end{abstract}

Le phénomène produit par la rencontre de la science, de l'éthique et du politique (qui est, comme le dit Max Weber, le règne de la "violence légitime»), tel qu'il s'observe aujourd'hui dans le domaine des soins de santé, est en quelque sorte une parfaite illustration du commentaire de Julien Freund, qui disai : "Avec la décision la violence s'introduit dans le monde». Et qui ajoutait: "Autant Max Weber a voulu que la pensée reste toujours ouverte au niveau du savoir, autant il a exigé qu'elle se ferme dans l'action par la responsabilité et la résolution ${ }^{29}$ ".

La norme éthique, comme certaines espèces en voie d'extinction, apparaît d'autant plus précieuse que son existence est perçue comme fragile dans le monde actuel. Dans la mesure où «l'éthique est l'investigation du sens de la vie, ou de ce qui rend la vie digne d'être vécue, ou de la façon correcte de vivre", l'interprétation de la norme éthique est liée à la question du sens ${ }^{30}$.

27. Cf. Max Weber, Le savant et le politique, op. cit., p. 77.

28. Ibid., p. 86-87.

29. Cf. Max Weber, Essais sur la théorie de la science, op. cit., p. 115.

30. Cf. Ludwig Wittgenstein, Leçons et conversations, suivies de Conférence sur l'éthique, Paris, Gallimard, 1971, p. 144. 
Toute norme éthique doit se risquer par conséquent dans les rapports que la pensée entretient avec l'action; et son destin dépend de la signification qui est alors donnée au jeu de l'ouverture et de la fermeture auquel songeait Max Weber, ce jeu sans lequel il n'est pas de liberté.

Pierre Gendron Centre de recherche en droit public Université de Montréal

"La sociologie, dit Wittgenstein, a le devoir de décrire aussi bien nos actions et les valeurs que nous attachons à ce qui nous entoure que celles des nègres (sic). Elle ne peut que relater ce qui arrive. Mais, dans la description du sociologue, la proposition: "telle ou telle chose signifie un progrès" ne doit jamais apparaître" (ibid., p. 157). Le point de vue de la philosophle, tel qu'il s'exprime notamment dans la Conférence surl'éthique, consiste plutôtà vouloir «abolir le savoir", comme disait Kant: "das Wissen aufheben" (um zum Glauben Platz zu bekommen). Sil'éthique est "ce grâce à quoi je suis capable de penser le bien et le mal» (Wittgenstein, op. cit., p. 173), la détermination de l'agir humain est aussi empreint de finitude. C'est pourquoi Schelling définissait le concept vivant de la liberté comme celui d'un pouvoir du bien et du mal ein Vermögen des Guten und des Bösen et expliquait qu'elle était même proprement, en tant que liberté ahumaine», un pouvoir pour le mal (zum Bösen), comme dans l'expression "pour le meilleur et pour le pire». Chez Max Weber, cela donne le «paradoxe des conséquences».

L'homme historique ne saurait fonder ses choix uniquement sur une éthique de la responsabilité qui dépend trop des moyens de prévision qu'il est possible de se donner et qui se heurte par conséquent à la limite de ce qui peut être constitué en objet de connaissance. À l'heure de la dissolution des grands récits idéologiques, l'éthique wébérienne de la politique nous rappelle que la "conviction" constitue un élément tout aussi essentiel de la conduite humaine que le "sentiment de la responsabilité» : voir Claude Piché, «Max Weber et le néo-kantisme : pour une politique de la modemité", Revue de Métaphysique et de Morale, 99 (1994), p. 327. 344. 